\title{
Discrimination between Onchocerca volvulus and 0 . ochengi filarial larvae in Simulium damnosum (s.l.) and their distribution throughout central Ghana using a versatile high-resolution speciation assay
}

Stephen R. Doyle ${ }^{1,5^{*}+}$, Samuel Armoo ${ }^{1,2+}$, Alfons Renz ${ }^{3}$, Mark J. Taylor ${ }^{4}$, Mike Yaw Osei-Atweneboana ${ }^{2}$ and Warwick N. Grant ${ }^{1}$

\begin{abstract}
Background: Genetic surveillance of the human filarial parasite, Onchocerca volvulus, from onchocerciasis endemic regions will ideally focus on genotyping individual infective larval stages collected from their intermediate host, Simuliid blackflies. However, blackflies also transmit other Onchocerca species, including the cattle parasite $O$. ochengi, which are difficult to distinguish from the human parasite based on morphological characteristics alone. This study describes a versatile approach to discriminate between $O$. volvulus and $O$. ochengi that is demonstrated using parasite infective larvae dissected from blackflies.

Results: A speciation assay was designed based on genetic differentiation between $O$. volvulus and $O$. ochengi mitochondrial genome sequences that can be performed in high-throughput high-resolution melt (HRM)- or lower throughput conventional restriction fragment length polymorphism (RFLP) analyses. This assay was validated on 185 Onchocerca larvae dissected from blackflies captured from 14 communities in Ghana throughout 2011-2013. The frequency of 0 . ochengi was approximately $67 \%$ of all larvae analysed, which is significantly higher than previously reported in this region. Furthermore, the species distribution was not uniform throughout the study region, with $25 \%, 47 \%$ and $93 \%$ of O. volvulus being found in the western-most (Black Volta, Tain and Tombe), the central (Pru) and eastern-most (Daka) river basins, respectively.

Conclusions: This tool provides a simple and cost-effective approach to determine the identity and distribution of two Onchocerca species, and will be valuable for future genetic studies that focus on parasites collected from blackflies. The results presented highlight the need to discriminate Onchocerca species in transmission studies, as the frequency of each species varied significantly between the communities studied.
\end{abstract}

Keywords: Onchocerciasis, Onchocerca volvulus, Onchocerca ochengi, High-resolution melt analysis (HRM), Restriction fragment length polymorphism (RFLP), Speciation, Ghana

\footnotetext{
* Correspondence: stephenrdoyle1@gmail.com

${ }^{\dagger}$ Equal contributors

'Department of Animal, Plant and Soil Sciences, La Trobe University,

Bundoora 3086, Australia

${ }^{5}$ current address: Wellcome Trust Sanger Institute, Wellcome Genome

Campus, Hinxton, Cambridgeshire CB10 1SA, UK

Full list of author information is available at the end of the article
} 


\section{Background}

Onchocerca volvulus is a human filarial parasite responsible for the disease onchocerciasis, and is transmitted between hosts by blackflies of the genus Simulium. Collection of blackflies throughout disease endemic regions forms a significant part of onchocerciasis surveillance campaigns to determine the prevalence and transmission of the parasite. Two approaches are commonly used to detect $O$. volvulus. The first approach, pool-screening, has been extensively used to determine the prevalence of a single parasite in a pool of low hundreds of blackfly heads [1], which can be detected using an Onchocercaspecific O-150 polymerase chain reaction (PCR) assay together with an $O$. volvulus-specific DNA probe. Poolscreening has been the predominant tool used for parasite surveillance by the African Programme for Onchocerciasis Control (APOC, Burkina Faso), and is suitable particularly when the prevalence of $O$. volvulus is low in areas where widespread use of ivermectin has taken place. Adaptation of the pool-screening approach to detect $O$. volvulus has recently been demonstrated using loop-mediated isothermal amplification (LAMP) targeting either the O. volvulus glutathione $S$-transferase 1a gene [2], or the O-150 repeat (Nankoberanyi, Doyle \& Grant, personal communication; [3]), which promises to improve the throughput, cost and sensitivity of the conventional approach. Although poolscreening has the capacity to examine large numbers of flies, the primary objective of the assay is simply to determine the presence or absence of the parasite in a defined number of vectors. The second approach to detect $O$. volvulus is via the dissection of larvae from individual blackflies. This method pre-dates the use of poolscreening (having been in use since the initial Onchocerciasis Control Programme (OCP) in West Africa) and is still practiced widely where access to pool-screening is problematic. Dissection has the advantages that it is done at the point of fly collection and does not require the relatively complex, multi-step protocol of O-150 pool screening, and allows the developmental stage and location of the parasite(s) within the blackfly to be determined [4]. Data on larval prevalence in the vector are used together with the biting density of the fly to calculate the transmission potential of the parasite [5, 6]. It has the disadvantage, however, that it is not possible to routinely differentiate between $O$. volvulus and other Onchocerca species that may be transmitted concurrently by the same flies.

Genetic diagnostic tools are under development by us and others that aim to genetically define parasite populations, characterise transmission zones, and screen parasite populations for polymorphisms associated with variation in response to ivermectin [7] as a means of better defining the source and likely impact on mass drug administration effectiveness of parasite transmission in areas in which parasite transmission continues despite ivermectin distribution [8-10], i.e. genetic epidemiology. Such genetic epidemiological analyses could be integrated with, and add value to, existing entomological surveillance practices using the vector stages of the parasite as a non-invasive source of parasite material rather than microfilaria obtained from skin snips or adult parasites obtained by nodulectomy. Genetic analysis of parasites dissected from flies for the purpose of genetic epidemiology will require an efficient and sensitive molecular means to determine the species of parasite collected.

Species characterisation of dissected larvae is challenging (although not impossible) based on morphological characteristics $[6,11,12]$; it requires significant time and expertise, and is therefore relatively low-throughput. O150-based diagnostic assays remain the predominant tool for molecular discrimination of $O$. volvulus from other Onchocerca species $[9,13]$, although sequencing of mitochondrial DNA derived amplicons has proven to be an effective approach to delineate the phylogeny of the genus Onchocerca [14]. However, many studies describe the prevalence of $O$. volvulus larvae in blackflies without molecular confirmation [15-18]. Given that a number of Onchocerca species, including the common cattle parasite $O$. ochengi, can be transmitted by the same vector [6], it is likely that $O$. volvulus prevalence may easily be overestimated, and in turn, negatively influence the predictions of disease transmission. In this study, we report the development of a versatile molecular tool to discriminate the onchocerciasis parasite $O$. volvulus from other Onchocerca species such as $O$. ochengi based on mitochondrial DNA (mtDNA) sequence variation. We were motivated to develop a new speciation tool, rather than using an existing O-150 assay, as a primary screening approach to discriminate $O$. volvulus from other Onchocerca larvae prior to high resolution melt (HRM) genotyping for population and other genetic epidemiological analyses. This approach was validated using Onchocerca larvae dissected from blackflies that were collected from 3 geographically distinct regions in Ghana.

\section{Methods}

Blackflies were sampled from 14 communities in 5 river basins in central Ghana during 2011 (number of blackflies $(n)=12,031), 2012(n=9,706)$ and $2013(n=9,138)$ using a human landing collection protocol as described previously [19]. Ethical approval was obtained for the use of human vector collectors from the Institutional Review Board of the Council for Scientific and Industrial Research, Ghana. All blackflies caught were dissected to collect Onchocerca larvae (129 from the head, 51 from the thorax, and 5 from the abdomen used here), after which all larvae collected were dried on microscope slides for preservation. In total, 160 infective (L3), together with 21 pre-infective L2 and 
4. L1 larvae were analysed (Table 1) using the molecular techniques described, which included 14 larvae collected from 8 infected flies in the 2011 cohort, 24 larvae from 11 flies collected in 2012, and 147 larvae from 110 flies collected in the 2013 cohort. Individual larva were recovered from slides by applying $2 \mu \mathrm{l}$ of HPLC-grade water (SigmaAldrich, Castle Hill, Australia) directly onto the specimen and allowing it to rehydrate, so that the larva would detatch from the slide or could be dislodged by a gentle scrape with a pipette tip. Each larva was individually transferred to $20 \mu$ of sample lysis solution [DirectPCR Lysis reagent (MouseTail; Viagen Biotech, Los Angeles, USA) supplemented with $0.3 \mathrm{mg} / \mathrm{ml}(\mathrm{w} / \mathrm{v})$ proteinase $\mathrm{K}$ (Roche, Mannheim, Germany)], and incubated for $18 \mathrm{~h}$ at $55^{\circ} \mathrm{C}$ followed by $85^{\circ} \mathrm{C}$ for $1 \mathrm{~h}$. Larval lysates were diluted with HPLCgrade water $1 / 10$ prior to PCR. Adult $O$. volvulus samples were obtained as previously described [10]. Adult $O$. ochengi were collected from infected cattle udders obtained from an abattoir in Ngaoundéré, Cameroon. Nodules were collected from the skin, from which worms were extracted using forceps via a small incision before being fixed in $95 \%$ ethanol. DNA was extracted from individual adult $O$. volvulus and O. ochengi samples using an Isolate II Genomic DNA extraction kit (Bioline, Sydney, Australia) following the manufacturers' instructions.

High resolution melt analysis was performed after real time PCR amplification of a 79-bp product using the Bio-Rad CFX system (Bio-Rad Laboratories, Hercules, USA). A $10 \mu$ reaction mix was prepared by combining $5 \mu \mathrm{l}$ of $2 \times$ SsoAdvanced Universal SYBR Green qPCR Supermix (Bio-Rad Laboratories), $0.5 \mu \mathrm{l}$ of each of SP_OVOO_79 bp (5'-GTT TGG TTC TTG TTG ATT TG-3') and ASP_OVOO_79 bp (5'-ACA TTA AAC CCC CTT ACC-3') primers (0.5 $\mu \mathrm{M}$ working concentration), $2 \mu \mathrm{l}$ of HPLC grade water, and $2 \mu \mathrm{l}$ of the diluted larva lysate. The amplification protocol was performed as follows: $98{ }^{\circ} \mathrm{C}$ for $3 \mathrm{~min}$, followed by 45 cycles of $98{ }^{\circ} \mathrm{C}$ for $10 \mathrm{~s}, 59.5{ }^{\circ} \mathrm{C}$ for $10 \mathrm{~s}$ and $72{ }^{\circ} \mathrm{C}$ for $10 \mathrm{~s}$. The melt protocol was performed immediately after the amplification, and was as follows: $95{ }^{\circ} \mathrm{C}$ for $10 \mathrm{~s}$, followed by a $65{ }^{\circ} \mathrm{C}$ annealing step from which the temperature was increased by $0.2{ }^{\circ} \mathrm{C} /$ plate read until a final temperature of $95{ }^{\circ} \mathrm{C}$. PCR products generated from adult O. volvulus and $O$. ochengi, as well as a subset of 5 larvae samples from each melt curve group (representing both $O$. volvulus and $O$. ochengi larvae), were cloned into the plasmid pGem-T-Easy (Promega, Madison, USA) and sequenced to confirm the nucleotide variants (Macrogen, Seoul, Korea). All lysates, together with positive (cloned products and adult $O$. volvulus and $O$. ochengi genomic DNA samples) and no-template controls (NTC), were analysed in duplicate. Raw melt data was analysed using Precision Melt software (version 1.2; Bio-Rad Laboratories), from which the data was exported into Microsoft Excel for further quantitative analysis. Spatial (within flies i.e. head, thorax or abdomen, and between river basins), temporal (sampling year) and larval stage (L1, L2 or L3) differences between $O$. volvulus and $O$. ochengi were each examined using a $2 \times 3$ Chi-square $\left(\chi^{2}\right)$ test with $2^{\circ}$ of freedom, and a $P$-value $<0.05$ was considered to represent significant deviation from the null hypothesis.

A subset of post-PCR and HRM products were analysed by ApaI restriction digest to confirm the O. volvulusspecific digestion. A $20 \mu \mathrm{l}$ reaction consisting of $10 \mu \mathrm{l}$ PCR product together with $0.5 \mu \mathrm{l}$ ApaI $(50,000 \mathrm{U} / \mathrm{ml}$; New England Biolabs, USA), $2 \mu$ l of CloneSmart buffer and $7.5 \mu \mathrm{l}$ of HPLC-grade water was incubated for $3 \mathrm{~h}$ at $25{ }^{\circ} \mathrm{C}$, after which the products were run on a $2 \%$ agarose gel at $100 \mathrm{~V}$ for 50 mins and visualised using GelRed DNA stain (Biotum, Freemont, USA).

\section{Results and discussion}

Whole mitochondrial genome alignments of the $O$. volvulus (NC_001861; [20]) and O. ochengi (obtained from genomic sequence available at http://www.nematodes.org/ genomes/onchocerca_ochengi/; Blaxter Lab, University of Edinburgh) sequences was performed to identify a PCR compatible region that contained (i) a restriction site that was unique to one species, and (ii) additional polymorphism(s) that would result in a difference in the melting temperature between amplicons generated for each of the 2 species. A 79-bp region within the nad1 gene spanning 8,655- to 8,733-bp of the O. volvulus mitochondrial genome was chosen that contained 2 synonymous and 1 non-synonymous nucleotide $\mathrm{C}>\mathrm{T}$ transitions (C8683T [Ala > Ala, C8693T [Leu > Phe], \& C8714T [Leu > Leu]) between the mtDNA alignment of the 2 species, 1 of which (C8693T; Leu > Phe) was found in an ApaI restriction site that is present in O. volvulus but absent in $O$. ochengi (Fig. 1a). A fourth discriminating variant was identified in this 79 bp region (G8659A; Leu > Leu); however, it was not ultimately used as it was located at the 5 ' end of the optimal forward primer binding site. Melt curve analysis demonstrated a shift in Tm between the 2 sequences, with a mean $\mathrm{Tm}$ of $79.8^{\circ} \mathrm{C}$ and $78.6{ }^{\circ} \mathrm{C}$ for the O. volvulus- and O. ochengi-derived sequences, respectively (Fig. 1b). HRM species determination of the unknown larval samples was performed by automated clustering of the unknown larval melt curves to curves derived from the control adult $O$. volvulus (Fig. 1b, c; green) and O. ochengi (Fig. 1b, c; red) samples. This translated into a significant and consistent deviation in melt curves between $O$. volvulus and $O$. ochengi samples as depicted in the fluorescence difference plots (Fig. 1c); these plots accentuate the difference in melt profiles throughout the temperature range (Fig. 1b) relative to a reference curve (set to $O$. ochengi adult control), and in turn, emphasizes differences between groups 
Table 1 Distribution of communities and samples used in this study

\begin{tabular}{|c|c|c|c|c|c|c|c|c|c|c|}
\hline \multirow[t]{3}{*}{ Community } & \multirow[t]{3}{*}{ River basin } & \multicolumn{2}{|c|}{ Geographical coordinates } & \multicolumn{6}{|c|}{ Larvae processed and species identified by HRM per sample collection year ${ }^{\mathrm{a}}$} & \multirow{3}{*}{$\begin{array}{l}\text { Total no. of } \\
\text { larvae per } \\
\text { community }\end{array}$} \\
\hline & & \multirow[t]{2}{*}{ Latitude } & \multirow[t]{2}{*}{ Longitude } & \multicolumn{2}{|c|}{2011 (12,031 flies dissected) } & \multicolumn{2}{|c|}{2012 (9,706 flies dissected) } & \multicolumn{2}{|c|}{2013 (9,138 flies dissected) } & \\
\hline & & & & O. volvulus & O. ochengi & O. volvulus & O. ochengi & O. volvulus & O. ochengi & \\
\hline Abua (ABA) & Pru & 7.58587 & -0.53485 & 1 & - & - & - & - & - & 1 \\
\hline Agbelekame I (AB) & Black Volta & 8.14018 & -2.12185 & 2 & 2 & 1 & 4 & 8 & $9\llcorner 2 ; 27$ L3; & 53 \\
\hline Akanyakrom (AKA) & Black Volta & 8.289 & -2.277 & - & - & 5 & - & - & - & 5 \\
\hline Asubende (ASU) & Pru & 8.01085 & -0.58528 & - & - & 2 & - & 5 & 2 & 9 \\
\hline Baaya (BAY) & Pru & 8.01119 & -0.59444 & - & - & - & - & 2 & $1 \mathrm{~L} 2 ; 12 \mathrm{~L} 3$ & 15 \\
\hline Beposo (BEP) & Pru & 8.0026 & -0.57395 & - & - & - & - & 1 & - & 1 \\
\hline Fawoman (FAW) & Pru & 8.0111 & -1.01303 & - & - & 1 & 2 & - & - & 3 \\
\hline Fawoman-Banda (FAB) & Tombe & 8.07108 & -2.1441 & - & - & - & 6 & 2 & - & 8 \\
\hline Kyingakrom (KYN) & Black Volta & 8.05578 & -2.03266 & - & 3 & - & - & - & - & 3 \\
\hline Mantukwa (MAN) & Pru & 8.01064 & -1.0014 & - & - & - & - & 2 & 2 & 4 \\
\hline New Longoro (NLG) & Black Volta & 8.08158 & -2.02096 & - & 1 & - & - & 1 & 1 L2; 8 L3 & 11 \\
\hline Senyase (SEN) & Pru & 8.01247 & -0.59251 & - & - & - & - & $1 \mathrm{~L} 1 ; 1 \mathrm{~L} 2 ; 1 \mathrm{~L} 3$ & - & 3 \\
\hline Tainso (TAN) & Tain & 8.0603 & -2.06538 & - & 5 & - & 3 & $1 \mathrm{~L} 1 ; 4 \mathrm{~L} 2 ; 7 \mathrm{~L} 3$ & 1 L1; 5 L2; 29 L3 & 55 \\
\hline Wiae (WIA) & Daka & 8.19219 & -0.09429 & - & - & - & - & $1 \mathrm{~L} 1 ; 12 \mathrm{~L} 3$ & 1 & 14 \\
\hline \multicolumn{4}{|c|}{ Total larvae processed per species per year } & 3 & 11 & 9 & 15 & 49 (3 L1; 5 L2; 41 L3) & 98 (1 L1; 16 L2; 81 L3) & 185 \\
\hline
\end{tabular}

a $\mathrm{L}$ larvae unless otherwise stated 
o. ochengi GTTTAGTTCTTGTTGATTTGCATCGGGCTCCTTTTGATTTTTCTGAGTGTGAAAGTGAGTTGGTAAGGGGGTTTAATGT

○. volvulus GTTTGGTTCTTGTTGATTTGCATCGGGCCCCTTTTGATCTTTCTGAGTGTGAAAGTGAGCTGGTAAGGGGTTTAATGT

b

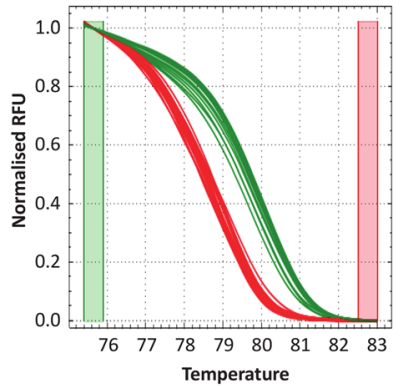

c

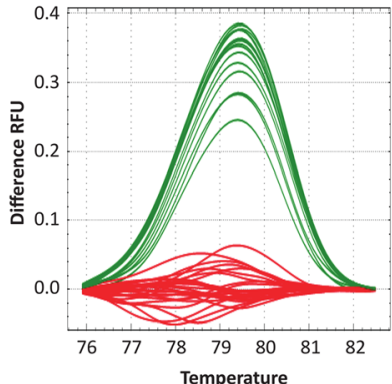

d

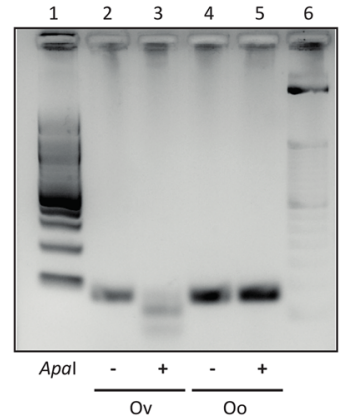

Fig. 1 Genetic discrimination of O. volvulus and O. ochengi. a Sequence of the PCR 79-bp amplicon used to discriminate between O. volvulus and 0 . ochengi. Nucleotide differences between the two species are highlighted in bold, and the Apal restriction site present in the $O$. volvulus sequence is underlined. $\mathbf{b}$ Normalised HRM melt curves from cloned positive control 79-bp products (pGem_Ov and pGem_Oo), adult O. volvulus and O. ochengi DNA, and 4 larvae samples from both species, each in duplicate. Curve colour of the larval samples is automatically determined based on the clustering of melt curves to the O. volvulus (green) and O. ochengi (red) adult samples. $\mathbf{c}$ The same samples are presented as in (b), showing HRM difference curves, which accentuate differences between the melt curve clusters in (b), normalised to the 0 . ochengi cluster. $\mathbf{d}$ Representative RFLP analysis of amplicons generated in the HRM assay for each species, showing digestion of the $O$. volvulus sequence, but not the $O$. ochengi sequence, with Apal

of sequences. To confirm that the difference between the 2 groups of melt curves were consistent with the prediction that the ApaI restriction site was present in the $O$. volvulus sequences but not in the $O$. ochengi sequences, 42 larvae-derived, 4 adult-derived and 2 cloned PCR products were analysed by restriction digest, of which a representative gel is shown in Fig. 1d. The melt curve and restriction digest data were $100 \%$ concordant in the samples analysed, demonstrating that both approaches were equally predictive of the species in question. Finally, cloned amplicons derived from both the adult control samples and 10 larvae (5 from each melt curve group) were analysed by Sanger sequencing, confirming the discriminating variants and concordance with the reference whole mitochondrial genome sequences for the 2 species. This result does not, however, exclude the possibility that additional genetic variation may exist within the HRMamplicon in either or both species of Onchocerca examined (or other potential Onchocerca species endemic to onchocerciasis regions [21-23]) that could result in melt curves that deviate from the O. volvulus and O. ochengi control sequences described here. For example, an analysis of the mitochondrial sequence of the $O$. ochengi Siisa variant [21] revealed a T,T,C haplotype that differed from the T,T,T haplotype of the adult $O$. ochengi presented (Dr Adrian Streit, personal communication). The melt temperature $(\mathrm{Tm})$ of this $O$. ochengi Siisa variant is predicted to be $79.4{ }^{\circ} \mathrm{C}$, compared to $79.7{ }^{\circ} \mathrm{C}$ for $O$. volvulus and $79.1{ }^{\circ} \mathrm{C}$ for $\mathrm{O}$. ochengi (Tm's were simulated using IDTDNA OligoAnalyser [https://www.idtdna.com/calc/ analyzer] using the following parameters: $0.5 \mathrm{uM}$ oligonucleotide, $50 \mathrm{mM} \mathrm{NaCl}$, and $3 \mathrm{mM} \mathrm{MgCl}$ concentrations), which if present would have generated a melt curve and Tm shift intermediate to the O. volvulus and $O$. ochengi control sequences used. Although a single base change is unlikely to confound the interpretation between the melt profiles of the $O$. volvulus and $O$. ochengi control samples described here, this assay does offer further opportunity beyond conventional O150 assays to explore genetic variation among and within species. HRM requires that any comparison of unknown samples be made against known positive control samples; therefore, to explore potential variation beyond the assay described here, amplicons that result in melt curves that do not cluster with controls must be sequenced to identify and confirm potential novel variation present.

Of the flies dissected, 166 contained Onchocerca larvae (Additional file 1: Table S1); only a single larva was recovered from each of 123 flies, whereas 43 flies contained multiple larvae (Additional file 1: Table S2; $25.90 \%$ of total; range: $2-6$ ). Of the flies from which larvae were processed by HRM (129 flies in total), 4 contained mixed species infections (Additional file 1: Table S2; 14.29 \% of flies with multiple larva, $3.10 \%$ of all flies processed), i.e. they contained both $O$. volvulus and $O$. ochengi larvae, which must represent larval uptake by the blackfly from 2 blood meals from different hosts at different times. This is not surprising, given the prevalence of cattle and human cohabitation, and that blackflies will seek a blood meal from either host [24, 25]. The overall 
proportion of each larval stage was similar between both species (Additional file 1: Table S3; $\chi^{2}=3.397, d f=2$, $P=0.091$ ), however, the spatial distribution of each stage within the fly was not equal; although a greater proportion of L3 were found in the head relative to the thorax in both species, the difference between both head and thorax was greatest in O. volvulus with a higher proportion of L3 collected from the head (Additional file 2: Figure S1, Additional file 1: Table S3; $\left.\chi^{2}=6.822, d f=2, P=0.016\right)$. This finding suggests that while the rate of development within the fly may be similar between the species, O. volvulus may migrate towards the head of the fly earlier that $O$. ochengi. These observations (co-infection and likely equivalent transmission potential [based on prevalence of L3]), do suggest that the human and bovine hosts are constantly exposed to both parasites which, in turn, raises an interesting question in regard to the number of times host-switching may have occurred between human and cattle (a speciation hypothesis whereby the most recent common ancestor of $O$. ochengi and $O$. volvulus was a cattle parasite that established in the human host [26]).

The distribution of both species throughout the sampling region was not uniform (Fig. 2). Although no temporal differences between sampling years for each species was seen (Additional file 1: Table S2: $X^{2}=1.075, d f$ $=2, P=0.292$ ), a significant spatial trend was observed that suggested that the western communities sampled had a lower prevalence of $O$. volvulus $(22.96 \%, n=135$; Black Volta, Tain and Tombe river basins), a roughly equal prevalence of $O$. volvulus and $O$. ochengi in the central Pru river basin $(47.22 \%$ O. volvulus; $n=36)$, and a higher prevalence of $O$. volvulus $(92.86 \%, n=14)$ in the eastern-most Daka river basin (Additional file 1:
Table S4; $\left.\chi^{2}=32.145, d f=2, P=5.234 \mathrm{e}^{-8}\right)$. These results are not necessarily in contrast to a recent investigation of persistent $O$. volvulus with negligible $O$. ochengi transmission in Ghana [9]. Much of that study was focused on 2 southern Ghanaian communities, and very few or no larvae were found in the 2 communities that were shared with this study (Asubende and Agborlekame, respectively); moreover, 3 of the 7 communities used by Lamberton et al. were east of the Black Volta Lake, which would be consistent with high $O$. volvulus prevalence observed in the eastern-most community (Wiae) sampled here. We speculate that the high infection rate reported and difference in prevalence of $O$. ochengi in a number of study regions presented here is correlated with the high numbers of cattle in the north-western river basins, particularly during the dry season (December to April). However, given that the sample size of larvae for many communities was low (median $=6.5$, range $=1-55$ larvae $/$ community; limited by the number of larvae present in the blackfly populations and therefore by the number of blackflies screened), further sampling is required to support these findings, particularly in the Daka river basin where Onchocerca larvae were obtained from only a single community out of the five communities in which flies were sampled.

\section{Conclusions}

A simple but versatile tool that discriminates between $O$. volvulus and $O$. ochengi single larvae obtained by dissection of the blackfly intermediate host is described. A HRM approach offers a number of advantages over traditional O-150-based assays for the speciation of larvae collected by dissection, including (i) the ability to detect and explore novel genetic variation, either within or between Onchocerca species, and (ii) a rapid, single tube

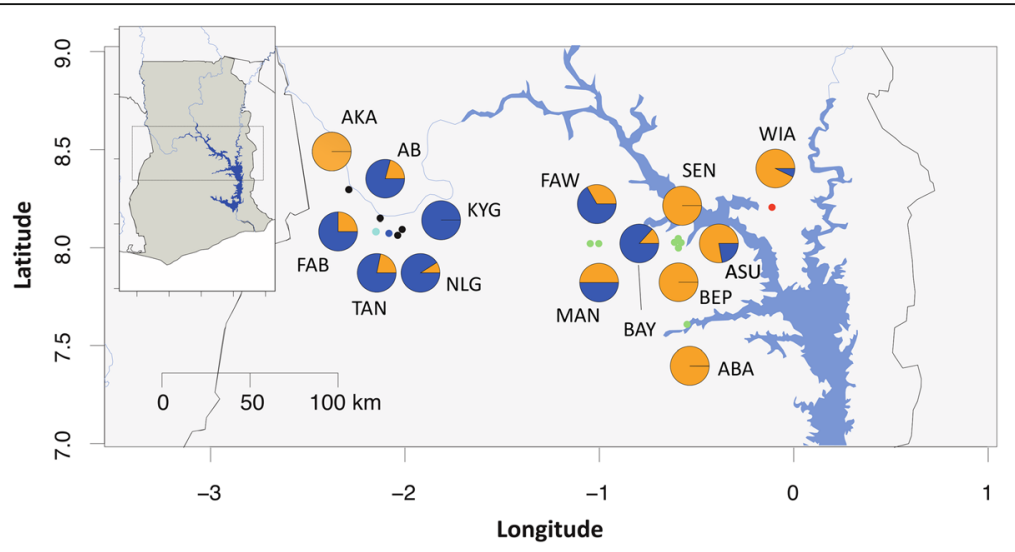

Fig. 2 Distribution of $O$. volvulus and O. ochengi throughout Ghana. Pie charts depict relative prevalence of O. volvulus (orange) and O. ochengi (purple) in each community. Communities sampled in central Ghana (see insert) and the number of samples analysed are as follows: Abua (ABA; $n=1)$, Agbelekame I (AB; $n=54)$, Akanyakrom (AKA; $n=5)$, Asubende (ASU; $n=9)$, Baaya (BAY; $n=15)$, Beposo (BEP, $n=1)$, Fawoman (FAW; $n=3)$, Fawoman-Banda ( $F A B, n=8)$, Kyingakrom (KYN; $n=3$ ), Mantukwa (MAN; $n=4)$, New Longoro (NLG; $n=11$ ), Senyase (SEN; $n=3)$, Tainso (TAN; $n=55)$, Wiae (WIA; $n=14)$. The colour coding of communities reflect the river basin in which they lie; Black Volta (black), Tain (dark blue), Tombe (light blue) Pru (green) and Daka (red) 
diagnostic assay format that can be run individually or in plate format (qPCR platform dependent). Note that this assay does not compete with the pool-screening O150 assay designed to detect single larvae in pools of blackflies but is designed as a component of a suite of genetic epidemiology tools under development in our laboratory to include "population of origin" and "likely ivermectin response phenotype" assays. By exploiting the multicopy nature of the mitochondrial genome (in the same way that O150 assays target a highly abundant repetitive element in the genome), this assay is very sensitive, requiring only 1 / 100th of a single larva, thus leaving sufficient material for further genetic epidemiological analysis. Although $O$. volvulus and $O$. ochengi samples could be distinguished easily via HRM or RFLP assays, no effort was made to identify other described Onchocerca species that may coexist in onchocerciasis endemic regions [21-23]; further work is required to characterise melt profiles and/or restriction sites from additional Onchocerca spp. voucher specimens or sequences as they become available. In the context of onchocerciasis entomological surveillance by dissection, which aims to detect transmission of the human pathogen, and the genetic epidemiological tools under development, this study demonstrates the need to accurately and efficiently determine the identity of larvae in the vector in order to correctly estimate parasite transmission. In Ghana, nationwide sampling of blackfly populations is necessary to determine the distribution of Onchocerca spp. Implementation of genetic tools such as those described here will inform the appropriate control strategy needed by onchocerciais control programmes as they strive towards onchocerciasis elimination in Ghana.

\section{Additional files}

Additional file 1: Table S1. Summary of microscope slides containing larvae dissected from blackflies, including location, date, life stage of larvae and location within the fly from which the larvae were dissected. Table S2. Summary data and analysis of larvae recovered and processed per blackfly, by species, community and geographic location. Table S3. Summary data and analysis of larvae collected per blackfly per year. Table S4. Summary data and analysis of larvae dissection from blackflies, by species, life stage ( $L 3, L 2$, or L1), and tissue (head, thorax, or abdomen). (XLSX $30 \mathrm{~kb}$ )

Additional file 2: Figure S1. Proportion of $O$. volvulus (orange) and $O$ ochengi (purple) larval life stages ( $\mathrm{L} 3, \mathrm{~L} 2, \& \mathrm{~L} 1)$ found during dissection of the blackfly head, thorax and abdomen. Values reported are a percentage of the total larvae found within each species. (TIF $60491 \mathrm{~kb}$ )

\section{Abbreviations \\ APOC: African Programme for Onchocerciasis Control; HRM: High-resolution melt; LAMP: Loop-mediated isothermal amplification; mtDNA: mitochondrial DNA; OCP: Onchocerciasis control programme; PCR: Polymerase chain reaction; RFLP: Restriction fragment length polymorphism; Tm: melt temperature}

\section{Acknowledgments}

We thank Mr. Francis Veriegh for contributing to the blackfly dissection, and PD Dr Adrian Streit, Dr Annette Kuesel and Dr Aime Adjami for helpful suggestions toward the manuscript. We also thank Dr Mark Blaxter and Dr Benjamin Makepeace for access to the unpublished O. ochengi genome sequence, which was generated with support of the EU-funded programme "Enhancing Protective Immunity Against Filariasis".

\section{Funding}

This investigation received financial support from TDR, the Special Programme for Research and Training in Tropical Diseases, co-sponsored by UNICEF, UNDP, The World Bank and WHO, the German Research Foundation (DFG Re 1536/5-1) and the European Foundation Initiative for Neglected Tropical Diseases. The funding bodies did not play any role in the design of the study, collection, analysis, interpretation of data or in writing of the manuscript.

\section{Availability of data and material}

All data analysed is presented in figures and tables within the manuscript and its additional files.

\section{Author's contributions}

SRD designed the study, performed the molecular biology and data analysis, and drafted the manuscript. MYO-A, MT and SA designed the entomological study for transmission assessment, SA was involved in the collection and dissection of blackflies, and preparation of larvae samples. MYO-A and MT was involved in the coordination of sample collection. AR provided samples. WG participated in the analysis and helped draft the manuscript. All authors read and approved the final manuscript.

\section{Competing interests}

The authors declare that they have no competing interests.

\section{Consent for publication}

Not applicable.

\section{Ethics approval and consent to participate}

Ethical approval was obtained for the use of human vector collectors from the Institutional Review Board of the Council for Scientific and Industrial Research, Ghana.

\section{Author details}

${ }^{1}$ Department of Animal, Plant and Soil Sciences, La Trobe University, Bundoora 3086, Australia. ${ }^{2}$ Council for Scientific and industrial Research, Water Research Institute, Accra, Ghana. ${ }^{3}$ Department of Comparative Zoology, Institute of Evolution and Ecology, University of Tübingen, Auf der Morgenstelle 28, 74074 Tübingen, Germany. ${ }^{4}$ Department of Parasitology, Liverpool School of Tropical Medicine, Liverpool, UK. ${ }^{5}$ Current address: Wellcome Trust Sanger Institute, Wellcome Genome Campus, Hinxton, Cambridgeshire CB10 1SA, UK

Received: 13 April 2016 Accepted: 4 October 2016

Published online: 10 October 2016

References

1. Katholi CR, Toe L, Merriweather A, Unnasch TR. Determining the prevalence of Onchocerca volvulus infection in vector populations by polymerase chain reaction screening of pools of black flies. J Infect Dis. 1995;172(5):1414-7.

2. Alhassan A, Makepeace BL, LaCourse EJ, Osei-Atweneboana MY, Carlow CK. A simple isothermal DNA amplification method to screen black flies for Onchocerca volvulus infection. PLOS ONE. 2014:9(10):e108927.

3. Alhassan A, Osei-Atweneboana MY, Kyeremeh KF, Poole CB, Li Z, Tettevi E, et al. Comparison of a new visual isothermal nucleic acid amplification test with PCR and skin snip analysis for diagnosis of onchocerciasis in humans. Mol Biochem Parasitol. 2016. doi: 10.1016/j.molbiopara.2016.07.006

4. Cheke RA, Garms R. Indices of onchocerciasis transmission by different members of the Simulium damnosum complex conflict with the paradigm of forest and savanna parasite strains. Acta Trop. 2013;125(1):43-52

5. Duke BO. Studies on factors influencing the transmission of onchocerciasis. V. The stages of Onchocerca volvulus in wild "forest" Simulium dannosum, the fate of the parasites in the fly, and the age-distribution of the biting population. Ann Trop Med Parasitol. 1968;62(1):107-16.

6. Eisenbarth A, Achukwi MD, Renz A. Ongoing transmission of Onchocerca volvulus after 25 years of annual ivermectin mass treatments in the Vina du 
Nord River Valley, in North Cameroon. PLoS Negl Trop Dis. 2016;10(2): e0004392.

7. Osei-Atweneboana MY, Eng JK, Boakye DA, Gyapong JO, Prichard RK. Prevalence and intensity of Onchocerca volvulus infection and efficacy of ivermectin in endemic communities in Ghana: a two-phase epidemiological study. Lancet. 2007;369(9578):2021-9.

8. Awadzi K, Boakye DA, Edwards G, Opoku NO, Attah SK, Osei-Atweneboana MY, et al. An investigation of persistent microfilaridermias despite multiple treatments with ivermectin, in two onchocerciasis-endemic foci in Ghana. Ann Trop Med Parasitol. 2004;98(3):231-49.

9. Lamberton PH, Cheke RA, Winskill P. Tirados I, Walker M, Osei-Atweneboana MY, et al. Onchocerciasis transmission in Ghana: persistence under different control strategies and the role of the simuliid vectors. PLoS Negl Trop Dis. 2015;9(4):e0003688.

10. Osei-Atweneboana MY, Awadzi K, Attah SK, Boakye DA, Gyapong JO, Prichard RK. Phenotypic evidence of emerging ivermectin resistance in Onchocerca volvulus. PLoS Negl Trop Dis. 2011;5(3):e998.

11. Wahl G, Schibel JM. Onchocerca ochengi: morphological identification of the L3 in wild Simulium damnosum s.l., verified by DNA probes. Parasitology. 1998;116(Pt 4):337-48.

12. McCall PJ, Townson H, Trees AJ. Morphometric differentiation of Onchocerca volvulus and O. ochengi infective larvae. Trans R Soc Trop Med Hyg. 1992; 86(1):63-5.

13. Adeleke MA, Mafiana CF, Sam-Wobo SO, Olatunde GO, Ekpo UF, Akinwale $\mathrm{OP}$, et al. Biting behaviour of Simulium damnosum complex and Onchocerca volvulus infection along the Osun River, Southwest Nigeria. Parasit Vectors. 2010;3:93.

14. Krueger A, Fischer P, Morales-Hojas R. Molecular phylogeny of the filaria genus Onchocerca with special emphasis on Afrotropical human and bovine parasites. Acta Trop. 2007;101(1):1-14.

15. Katabarwa MN, Eyamba A, Nwane P, Enyong P, Kamgno J, Kuete T, et al. Fifteen years of annual mass treatment of onchocerciasis with ivermectin have not interrupted transmission in the west region of Cameroon. J Parasitol Res. 2013;2013:420928

16. Katabarwa MN, Eyamba A, Nwane P, Enyong P, Yaya S, Baldiagai J, et al. Seventeen years of annual distribution of ivermectin has not interrupted onchocerciasis transmission in North Region, Cameroon. Am J Trop Med Hyg. 2011;85(6):1041-9

17. Opara KN, Fagbemi OB, Ekwe A, Okenu DM. Status of forest onchocerciasis in the Lower Cross River basin, Nigeria: entomologic profile after five years of ivermectin intervention. Am J Trop Med Hyg. 2005;73(2):371-6.

18. Wanji S, Kengne-Ouafo JA, Esum ME, Chounna PW, Tendongfor N, Adzemye $\mathrm{BF}$, et al. Situation analysis of parasitological and entomological indices of onchocerciasis transmission in three drainage basins of the rain forest of South West Cameroon after a decade of ivermectin treatment. Parasit Vectors. 2015;8(1):202

19. Walsh JF, Philippon B, Henderickx JE, Kurtak DC. Entomological aspects and results of the Onchocerciasis Control Programme. Trop Med Parasitol. 1987; 38(1):57-60

20. Keddie EM, Higazi T, Unnasch TR. The mitochondrial genome of Onchocerca volvulus: sequence, structure and phylogenetic analysis. Mol Biochem Parasitol. 1998;95(1):111-27.

21. Eisenbarth A, Ekale D, Hildebrandt J, Achukwi MD, Streit A, Renz A. Molecular evidence of 'Siisa form', a new genotype related to Onchocerca ochengi in cattle from North Cameroon. Acta Trop. 2013;127(3):261-5.

22. Wahl G. Identification of a common filarial larva in Simulium damnosum s.l. (type D, Duke, 1967) as Onchocerca ramachandrini from the wart hog. J Parasitol. 1996;82(3):520-4.

23. Wahl G, Renz A. Transmission of Onchocerca dukei by Simulium bovis in North-Cameroon. Trop Med Parasitol. 1991;42(4):368-70.

24. Achukwi MD, Harnett W, Renz A. Onchocerca ochengi transmission dynamics and the correlation of $\mathrm{O}$. ochengi microfilaria density in cattle with the transmission potential. Vet Res. 2000;31(6):611-21.

25. Lamberton P, Cheke RA, Walker M, Winskill P, Osei-Atweneboana MY, Tirados I, et al. Onchocerciasis transmission in Ghana: biting and parous rates of host-seeking sibling species of the Simulium damnosum complex. Parasit Vectors. 2014;7(1):511.

26. Morales-Hojas R, Cheke RA, Post RJ. Molecular systematics of five Onchocerca species (Nematoda: Filarioidea) including the human parasite, $\mathrm{O}$. volvulus, suggest sympatric speciation. J Helminthol. 2006:80(3):281-90.

\section{Submit your next manuscript to BioMed Central and we will help you at every step:}

- We accept pre-submission inquiries

- Our selector tool helps you to find the most relevant journal

- We provide round the clock customer support

- Convenient online submission

- Thorough peer review

- Inclusion in PubMed and all major indexing services

- Maximum visibility for your research

Submit your manuscript at www.biomedcentral.com/submit
Biomed Central 